\title{
LETTER
}

\section{Probiotics: an obedient ally or an insidious enemy?}

\author{
Metaxia N Papanikolaou*, Margarita Balla, Theonymfi Papavasilopoulou, Georgios Kofinas and Stylianos Karatzas
}

The first case of bloodstream infection by Pediococcus pentosaceus after the administration of a symbiotic formula to a critically ill patient is presented. The infection was successfully treated with ceftriaxone.

Probiotics are increasingly used in the intensive care unit (ICU), mainly for the management of intractable diarrhea. As probiotics are living organisms, one risk following their administration is infection of the host, primarily by Lactobacillus rhamnosus or Saccharomyces boulardii [1]. This is the first report of a bloodstream infection by Pediococcus pentosaceus following symbiotic administration to a critically ill patient.

A 64-year-old man with severe acute gallstone pancreatitis was admitted to our ICU; he had an APACHE II (Acute Physiology and Chronic Health Evaluation II) score of 35 and a Ranson score of 7. He stayed in the ICU for 191 days and eventually died of multiorgan failure. During his stay, he developed recurrent intra-abdominal abscesses for which he required both percutaneous drainage and surgical debridement. He was given total parenteral nutrition because of persistent diarrhea. The stool cultures were negative for Clostridium difficile toxin. As all other measures to control the diarrhea failed, we administered a probiotic-prebiotic combination on day 43. This was a formula with $4 \times 10^{10}$ bacteria once daily: $10^{10}$ colony-forming units of each of $P$. pentosaceus, Leuconostoc mesenteroides, L. paracasei, and L. plantarum and also $2.5 \mathrm{~g}$ of inulin, oat bran, pectin, and resistant starch (Synbiotic 2000 Forte; Medifarm, Kagerod , Sweden). On day 45 , the patient became septic and had a temperature of $39^{\circ} \mathrm{C}$, a white blood cell count of $14,350 /$ $\mathrm{mm}^{3}$, and a C-reactive protein of $100 \mathrm{mg} / \mathrm{dL}$. Blood cultures revealed the presence of $P$. pentosaceus (VITEK II; bioMérieux, Marcy l'Etoile, France) susceptible to penicillin G, ampicillin, ceftriaxone, erythromycin, and daptomycin. The probiotic was discontinued, and ceftriaxone was started. The patient responded to treatment and improved gradually. We believe that the bacteremia was triggered by the probiotic formula.

${ }^{*}$ Correspondence: xenia-pap@hotmail.com

Intensive Care Unit, Hippokrateion General Hospital of Athens, 114, Vas. Sophias Ave., 11521 Athens, Greece
Most probiotics can be safely used in patients receiving nutritional support. The formula that we administered has been extensively used before, and to the best of our knowledge, there were no reports relating this formula to infectious complications [2]. Nonetheless, in a recent clinical trial, an unexpected increase in mortality in probiotic-treated patients with severe pancreatitis was found; this increase cannot be ignored and calls into question the safety of probiotics [3].

Bloodstream infections by probiotics can be attributed to translocation across gut mucosa, to central venous catheter $(\mathrm{CVC})$ contamination, or to massive colonization [4]. In our case, translocation seems the most likely hypothesis; the tip of the CVC was sterile, and cultures from different sites (bronchial secretions, peritoneal fluids, and urine) were all negative. We conclude that P. pentosaceus bacteremia could be an emerging threat in critically ill patients receiving symbiotic formulas, despite their acceptable safety profile.

\section{Abbreviations}

CVC, central venous catheter; ICU, intensive care unit.

\section{Competing interests}

The authors declare that they have no competing interests.

Published: 5 November 2012

\section{References}

1. Whelan K, Myers CE: Safety of probiotics in patients receiving nutritional support: a systematic review of case reports, randomized control trials, and nonrandomized trials. Am J Clin Nutr 2010, 91:687-703.

2. Kotzampassi K, Giamarellos-Bourboulis E, Voudouris A, Kazamias P, Eleftheriadis E: Benefits of a symbiotic formula (Synbiotic 2000 forte) in critically ill trauma patients: early results of a randomized controlled study. World J Surg 2006, 30:1848-1855.

3. Morrow LE, Gogineni V, Malesker MA: Synbiotics and probiotics in the critically ill after the PROPRATRIA trial. Curr Opin Clin Nutr Metab Care 2012, 15:147-150.

4. Lolis N, Veldekis D, Moraitou H, Kanavaki S, Velegraki A, Triandafyllidis C, Tasioudis C, Pefanis A, Pneumaticos I: Saccharomyces boulardii fungemia in an intensive care unit patient treated with caspofungin. Crit Care 2008, 12:414.

\section{doi:10.1186/cc11806}

Cite this article as: Papanikolaou MN, et al.: Probiotics: an obedient ally or an insidious enemy? Critical Care 2012, 16:456. 